\title{
Premiers éléments de caractérisation de la morphodynamique de deux plages de la mer d'Iroise
}

\author{
Aurélie Dehouck $^{(\mathrm{a})}$, Véronique $\mathrm{Cuq}^{(\mathrm{b})}$, \\ Bernard Fichaut $^{(\mathrm{c})}$, Serge Suanez ${ }^{(\mathrm{c})}$ \\ (a) doctorante,(b) ingénieur d'étude UBO,(c) maître de conférence UBO \\ Laboratoire GEOMER, UMR 6554 LETG, Institut Universitaire Européen de la Mer \\ 29280 Plouzané Aurelie.Dehouck@univ-brest.fr
}

\section{Résumé}

Ce papier fait le point sur les premiers résultats issus du suivi à haute fréquence des plages sableuses de la mer d'Iroise entrepris en 2003. La démarche s'appuie sur une analyse géostatistique (en composantes principales) de la variabilité des profils de plage à Porsmilin et Corsen conduite en parallèle avec l'étude des conditions hydrodynamiques.

\begin{abstract}
$\underline{\text { Abstract }}$
This paper deals with the first results of the high frequency monitoring of sand beaches in west Brittany for the year 2003. The method lies on the study of beach profile variability using eigenfunctions analysis within the hydrodynamic forcings.
\end{abstract}

Mots-clés: Morphodynamique, plage sableuse, tempête, bilan sédimentaire, analyse en composantes principales.

\section{Introduction}

Dans le cadre de l'Observatoire du Domaine Côtier (ODC) de l'Institut Universitaire Européen de la Mer, un suivi morphosédimentaire à haute fréquence des estrans sableux de la mer d'Iroise a été entrepris à partir de janvier 2003 par le laboratoire Geomer - LETG UMR6554 CNRS. Ce travail consiste à mesurer les changements de profils transversaux de plage à une fréquence hebdomadaire à bi-hebdomadaire, suivant les conditions météorologiques.

La problématique sous-jacente à ce suivi long terme concerne l'enregistrement d'un signal des variations climatiques à partir de changements morphosédimentaires des plages observées. A partir de là, deux objectifs ont été définis : le premier consistant à étudier l'impact des forçages climatiques sur les modifications morphologiques et sédimentaires des plages, le second à modéliser le fonctionnement morphosédimentaire et dynamique des environnements côtiers retenus.

Cet article met l'accent sur le premier point. L'acquisition de plus d'un an d'enregistrement sur deux plages au contexte morphodynamique différent, permet de présenter des premiers résultats. Tout d'abord, nous décomposerons un "premier signal" des variations climatiques à court terme à partir de l'évolution saisonnière du bilan sédimentaire ; et dans un second temps, nous analyserons la variabilité spatio-temporelle des profils transversaux des plages.

\section{Présentation des sites d'étude}

Dans le cadre de cette étude, seules les plages de Corsen et de Porsmilin (figure 1) sont présentées parmi celles faisant l'objet du suivi morphologique de l'ODC. Le choix des sites 
d'observation repose sur deux éléments : leur exposition aux agents de forçages climatiques et météorologiques et leur contexte morphodynamique.

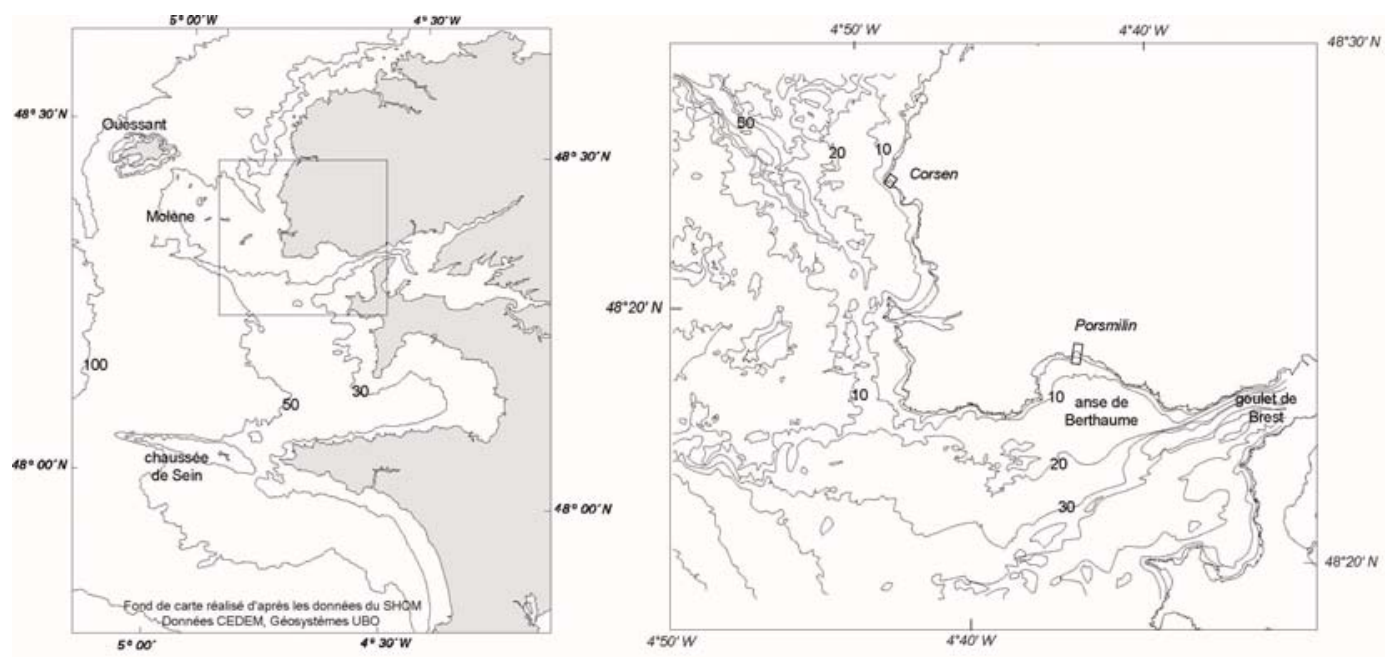

Figure 1 Bathymétrie de la mer d'Iroise d'après les données SHOMet carte de localisation des sites.

\subsection{Porsmilin}

Située dans l'anse de Berthaume, à l'entrée du goulet de Brest, la plage de Porsmilin bénéficie d'une situation abritée. Elle est principalement soumise aux flux océaniques de $\mathrm{S}$ $\mathrm{SW}$; toutefois, l'élargissement du plateau continental dans ce secteur, et surtout la présence de la chaussée de Sein, plus au sud, jouent un rôle important dans l'amortissement de la houle.

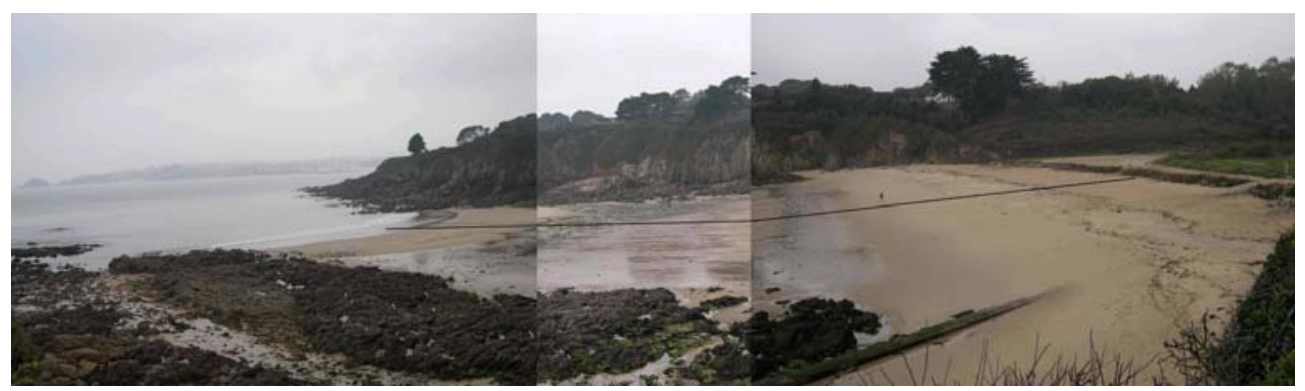

Figure 2 Plage de Porsmilin (anse de Bertheaume) le 13 octobre 2003et localisation du profil transversal.

La plage de Porsmilin (figure 2) correspond à une plage de fond d'anse constituée de sables moyens (médiane à $300 \mu \mathrm{m}$ ). Elle est formée d'un estran découvrant sur plus de $200 \mathrm{~m}$ en marée de vive-eau (marnage 6,5 $\mathrm{m}$ ), marqué par une faible pente $(\tan b=0.04)$. Toutefois, l'estran présente un haut de plage plus réfléchissant $(\operatorname{tanb}=0.06)$ toute l'année. Ce dernier est d'ailleurs caractérisé par la présence quasi permanente de croissants de plage. Le haut de plage est fermé par un remblai sur lequel un parking a été aménagé. Au pied de ce remblai, on observe la présence de dunes embryonnaires et de sables soufflés.

\subsection{Corsen}




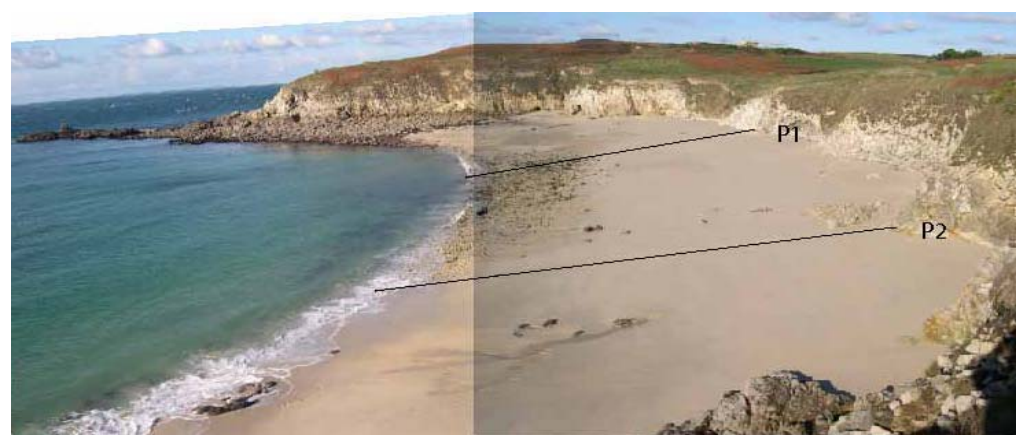

Figure 3 Plage de Corsen le 24 novembre 2003 et localisation des profils topométriques

La plage de Corsen se situe sur la côte ouest du Finistère, au sud de la pointe de Corsen (fig.1). Elle est principalement exposée aux flux océaniques de SW et d'ouest qui sont considérablement atténués par l'archipel de Molène et l'île de Ouessant. C'est une plage de poche cernée par des falaises aux escarpements sub-verticaux réfléchissants. Cette disposition explique l'absence d'un domaine supralittoral dunaire. Elle est uniquement formée d'un estran très court $(50$ à $60 \mathrm{~m})$ et très pentu $(\operatorname{tanb}=0.12)$. Les sables sont plus grossiers qu'à Porsmilin (médiane à $500 \mu \mathrm{m}$ ); ils recouvrent un cordon de galets qui affleure en bas d'estran.

\section{Méthodologie}

La mesure des variations morphosédimentaires des plages est réalisée à partir d'un tachéomètre laser le long de profils perpendiculaires au trait de côte. Un seul profil a été mis en place à Porsmilin (fig.2) car la plage fonctionne de façon similaire d'un bout à l'autre de l'estran. A l'inverse, deux profils ont été installés sur la plage de Corsen (fig.3), car, comme nous le verrons, aux variations transversales de l'estran s'ajoutent des déplacements sédimentaires longitudinaux. A Porsmilin et Corsen, les levés topographiques ont été opérés indifféremment selon les coefficients de marée conduisant à des longueurs de profil très variables au cours de l'année 2003.

Le traitement statistique des données topométriques repose sur une analyse en composantes principales $(\mathrm{ACP})$ de la matrice à une dimension horizontale constituée par les élévations du profil selon un pas régulier et par les dates de levé. Le but est d'extraire de nouvelles fonctions représentatives de la variabilité des profils lesquelles permettent d'expliquer les échanges sédimentaires intrasèques au profil en conjonction avec les forçages météo-marins. La démarche adoptée est abondamment documentée dans la littérature 1, 2, 3.

Les données d'élévation (figures 4 et 5) sont rééchantillonnées selon un pas constant en $\mathrm{x}$ ( $2.5 \mathrm{~m}$ et $1 \mathrm{~m}$ pour Porsmilin et Corsen respectivement). La distance transversale de l'ensemble des profils est arrêtée arbitrairement. La nécessité de devoir comparer l'information brute et commune à tous les profils amène à occulter le bas de plage de l'analyse. Les tableaux 1 et 2 présentent les fonctions issues de la combinaison linéaire des anciennes variables expliquant près de 95 et 98 \% de la variabilité des profils de plage à Porsmilin et Corsen.

\begin{tabular}{|c|c|c|c|}
\hline Composante & $\begin{array}{c}\text { Valeur } \\
\text { propre }\end{array}$ & $\begin{array}{c}\% \\
\text { variance }\end{array}$ & $\begin{array}{c}\text { \% variance } \\
\text { cumulée }\end{array}$ \\
\hline F1 & 34.9 & 62.4 & 62.4 \\
\hline F2 & 8.5 & 15.2 & 77.6 \\
\hline F3 & 5.7 & 10.1 & 87.8 \\
\hline F4 & 3.7 & 6.6 & 94.4 \\
\hline
\end{tabular}

\begin{tabular}{|c|c|c|c|}
\hline Composante & $\begin{array}{c}\text { Valeur } \\
\text { propre }\end{array}$ & $\begin{array}{c}\% \\
\text { variance }\end{array}$ & $\begin{array}{c}\text { \% variance } \\
\text { cumulée }\end{array}$ \\
\hline G1 & 25.6 & 50.3 & 50.3 \\
\hline G2 & 18.8 & 36.9 & 87.2 \\
\hline G3 & 3.9 & 7.8 & 95.0 \\
\hline G4 & 1.3 & 2.6 & 97.6 \\
\hline
\end{tabular}

Tableaux 1 et 2. Variances individuelles et cumulées associées aux nouvelles composantes issues de l'analyse en composantes principales des profils de Porsmilin (tableau 1) et de Corsen (tableau 2). 


\section{Analyse descriptive des variations des profils}

\subsection{Porsmilin}

Dans le détail, les profils fluctuent entre deux modes morphologiques stables: l'un estival (stade intermédiaire "low tide terrace ${ }^{4}$ ) avec une berme très construite et une plage dissipative, l'autre hivernal avec une plage aplanie accueillant ponctuellement une barre sableuse mobile (figure 4A). Les indices $\Omega^{4,5,6}$ et $\mathrm{K}^{7}$ aident à la caractérisation morphodynamique de la plage $e^{4,5}, \varepsilon^{8}$ et $\xi^{9}$, à celle de la dynamique de la zone de surf. Pour le profil hivernal, $\varepsilon$ et $\xi$ sont caractéristiques d'une plage de type intermédiaire ${ }^{4}(\varepsilon=9$ et $\xi \mathrm{b}=0.58$ ) et de déferlements type plongeant et déversant. Sur le profil d'été, on distingue le passage d'un domaine intermédiaire voire dissipant matérialisé par la terrasse $(\tan \beta=0.03$, $\varepsilon=44, \xi=0.27)$ à un domaine réfléchissant $(\tan \beta=0.06, \varepsilon=1.35, \xi=1.53)$ constitué par la berme (fig. 2 et 4 ).
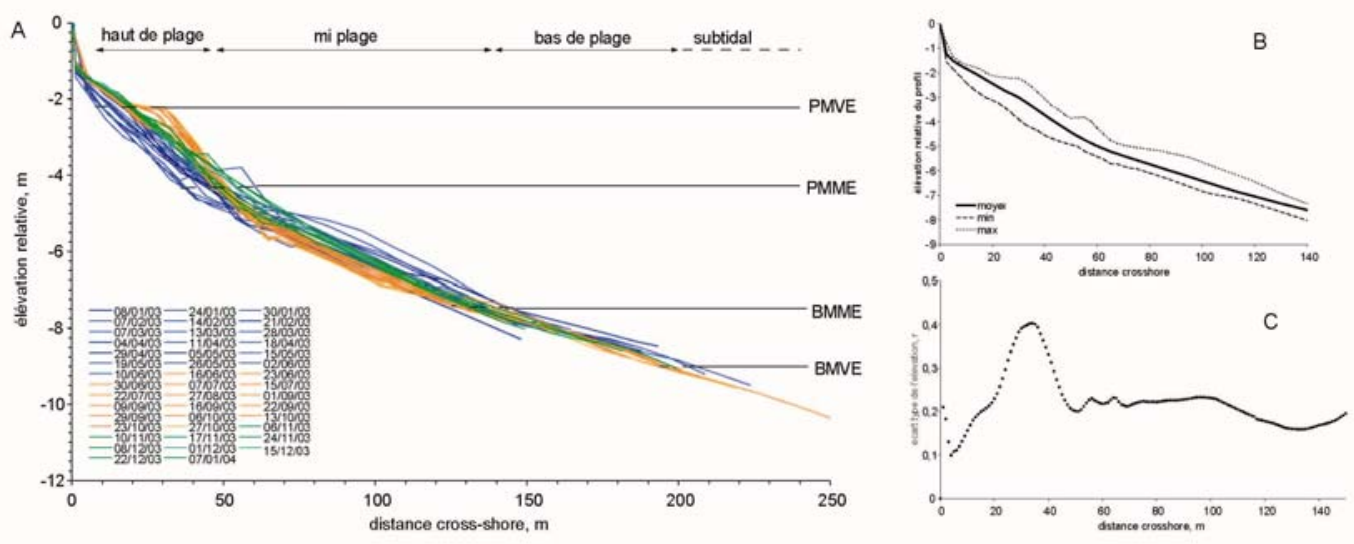

Figure 4 (A) Profils de plage à Porsmilin pour l'année 2003, (B) Profil moyen de la plage de Porsmilin, enveloppes maximale et minimale, $(\boldsymbol{C})$ écart-type de l'élévation fonction de la distance transversale.

La figure $4 \mathrm{~B}$ ci-dessus expose les enveloppes minimale, moyenne et maximale des profils à Porsmilin. Ce ne sont pas des profils réels pour des situations précises mais les enveloppes extraites de l'ensemble des données. On observe également l'écart-type des élévations selon la distance transversale10. La variabilité sur le profil est la plus grande 30 à $40 \mathrm{~m}$ après la tête de profil c'est à dire en haut de plage où se construit et s'efface la berme suivant les conditions météomarines.

\subsection{Corsen}

La figure 5 montre que la variabilité morphosédimentaire est forte sur l'ensemble du profil 2 tandis qu'elle est localisée au haut d'estran pour le profil 1. 


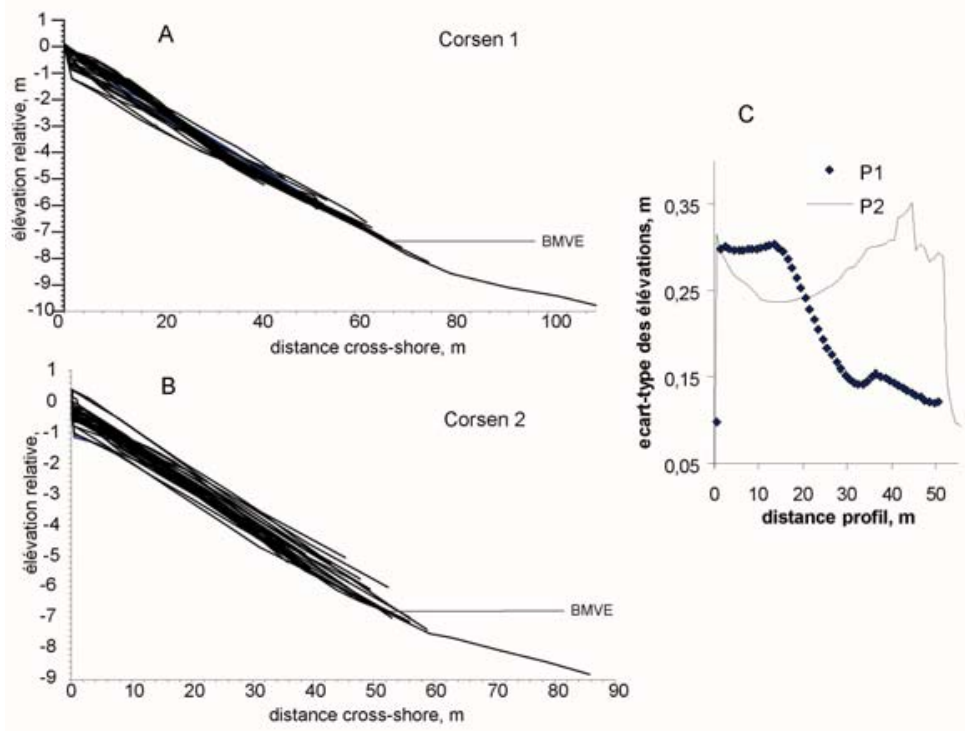

Figure 5 Profils de plage à Corsen pour l'année 2003, Corsen1 (A) et Corsen2 (B) correspondent aux profils nommés P1 et P2 sur la figure 5; (C) écart-type des élévations le long du profil pour les profils 1 et 2.

\section{$\underline{\text { 5.Bilans sédimentaires }}$}

Les bilans sédimentaires cumulés non nuls des plages de Porsmilin et Corsen (figures 6 et 7) montrent que les estrans ne sont pas des systèmes fermés et conservatifs à l'échelle de la variabilité annuelle.

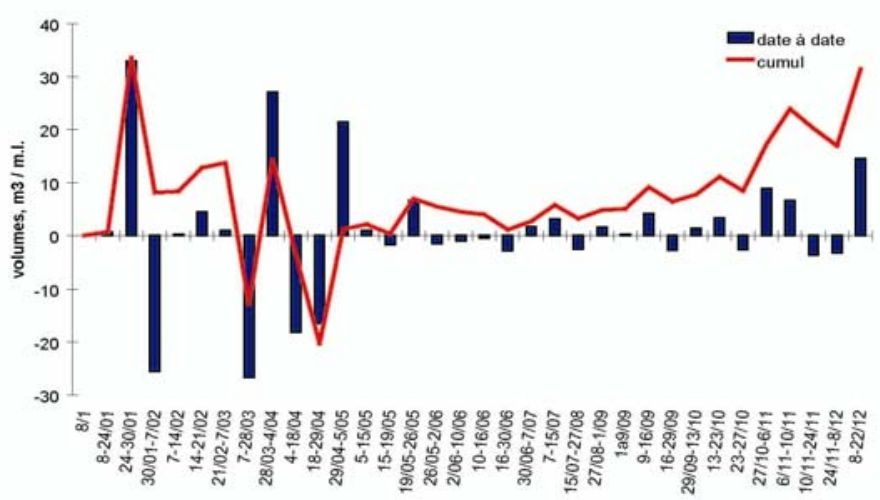

Figure 6 Bilans sédimentaires volumiques à Porsmilin au cours de l'année 2003

La variabilité volumique du système estran $(0-140 \mathrm{~m})$ à Porsmilin est forte de janvier à avril 03 et négligeable de mai à octobre 03. Malgré le retour des premiers coups de vent fin octobre (notés O1, N1 fig.9), la plage gagne en sédiment, ce qui va à l'encontre des schémas conventionnels du comportement saisonnier des plages sableuses. 

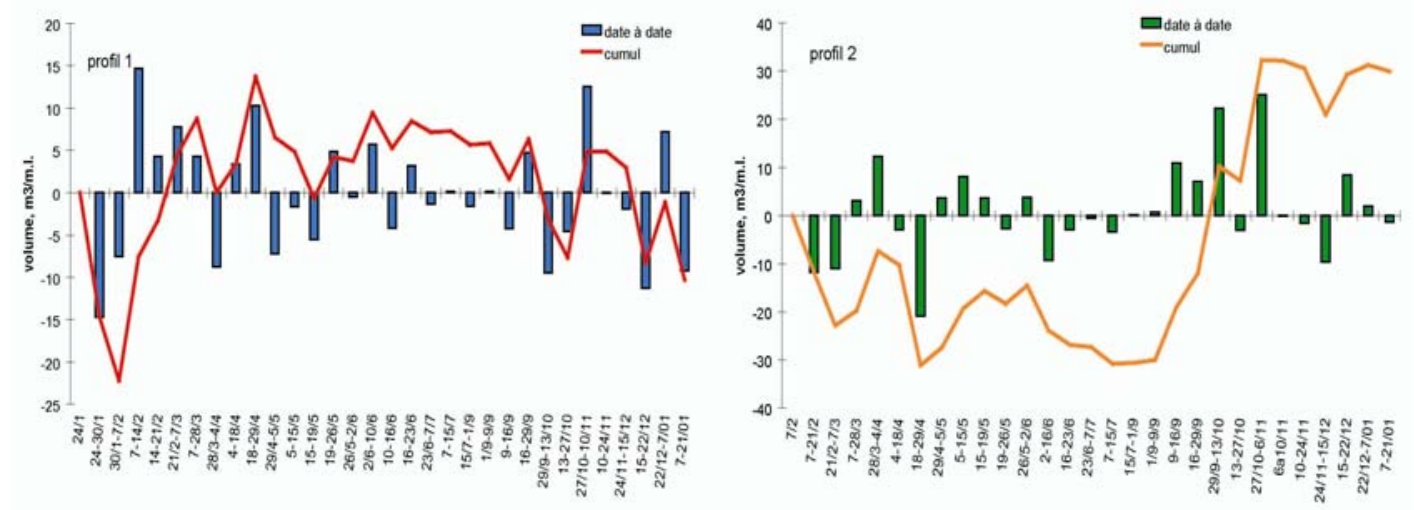

Figure 7 Bilans sédimentaires volumiques relatifs des profils 1 et 2 à Corsen au cours de l'année 2003

A Corsen, il existe un transit longitudinal fort du fait de l'obliquité de la houle incidente. Les bilans sédimentaires d'un profil à l'autre (figure 7) sont très variables et régulièrement opposés entre deux levés. Ainsi, de février à octobre, le bilan est globalement négatif sur le profil 2, le système regagnant du sédiment à partir des premiers coups de vent de l'hiver 2003/04. A l'inverse, le système est plus oscillant sur le profil 1, déficitaire pendant l'automne et l'hiver, et positif printemps-été 03 .

Cette divergence d'évolution des bilans sédimentaires entre les deux profils est liée aux transferts longitudinaux au sein de la plage. Ceux-ci semblent liés à une grande prépondérance des vents de secteur nord à partir d'octobre 2003 qui auraient favorisé l'engraissement du profil 2 au détriment du profil le plus au nord.

\section{Analyse en composantes principales des profils}

\subsection{Porsmilin}

Les résultats de l'ACP des profils transversaux à Porsmilin mettent en évidence la dynamique et les mouvements sédimentaires de l'estran. Seules deux fonctions F1 et F2 représentant $78 \%$ de la variabilité sont illustrées en figure 8. F1 est concernée par les échanges entre le haut de plage et la mi-plage tandis que F2 illustre les échanges intra-haut de plage et depuis la mi-plage vers le bas de plage.

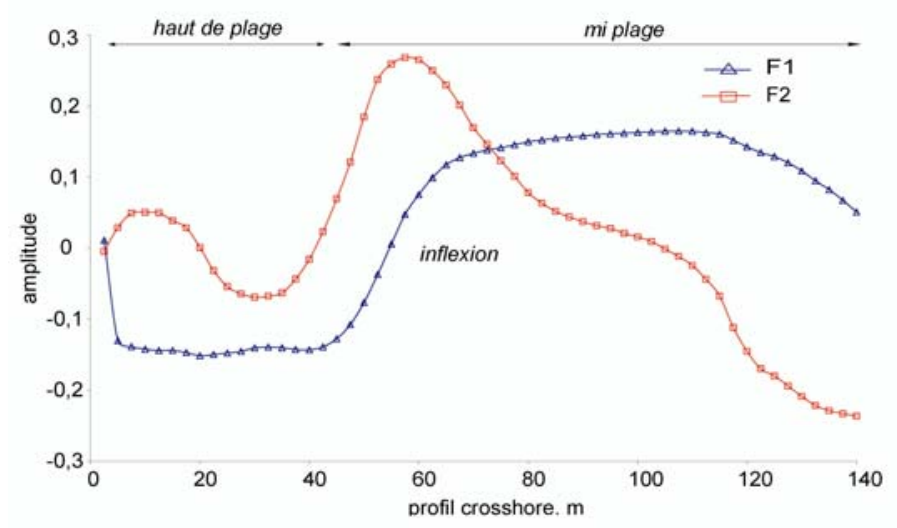

Figure 8 Composantes F1 et F2 fonction des anciennes variables (distance cross-shore) 
A partir de la connaissance du comportement qualitatif des fonctions F1 et F2 dans la dimension transversale (figure 8), les fluctuations de ces deux mêmes fonctions pour l'année 2003 revêtent une empreinte fortement saisonnière (figure 9). Lorsque, entre deux dates, l'amplitude de la fonction F1 croît, le profil de plage s'aplanit, le haut de plage démaigrit et les sédiments sont distribués sur la mi-plage. Ce sont typiquement les événements de tempête (notés J1, F3 à F6...), destructeurs, qui érodent le haut de plage et façonnent la plage vers un " profil d'hiver " (fig.9A). Au contraire, lorsque l'amplitude de F1 décroît, la tendance observée est à l'engraissement de la berme et à l'érosion du reste de l'estran lequel s'apparente à une terrasse à basse mer (fig.9B). A l'échelle annuelle, le profil évolue entre ces deux situations saisonnières, les tempêtes contribuant à son érosion, et les houles de beau temps à sa reconstruction et à l'accumulation des sédiments en haut de plage.

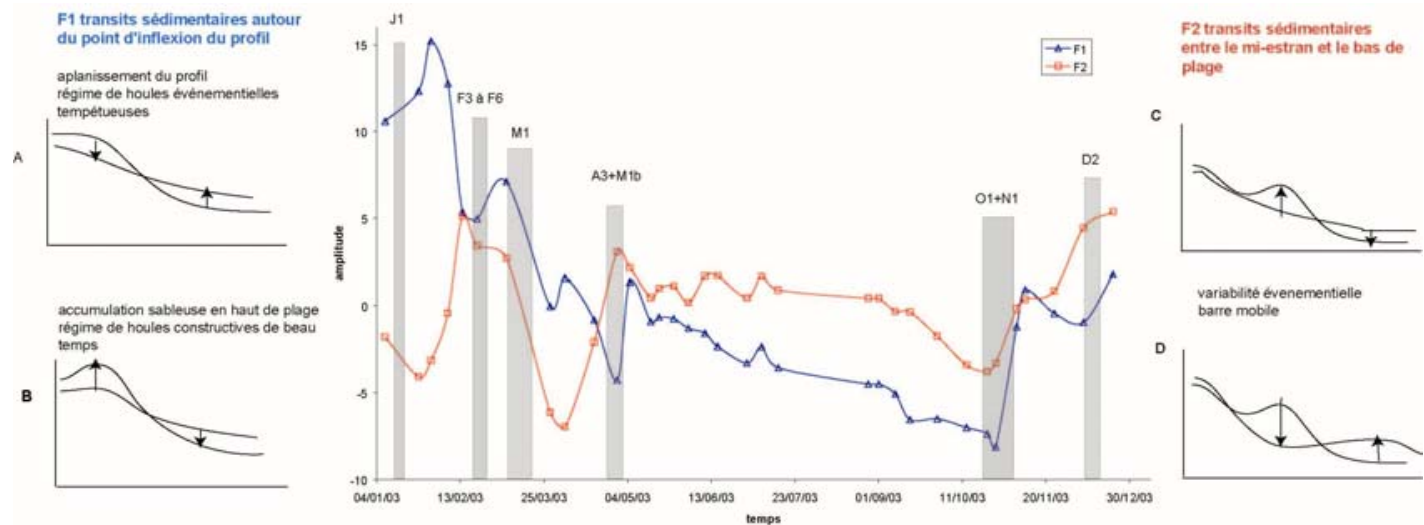

Figure 9 Composantes F1 et F2 à l'échelle temporelle (individus) issues de l'ACP des données cross-shore $x$ temporel à Porsmilin et schémas conceptuels du comportement de la plage selon F1 (schémas A, B) et F2 (C, D). Les événements notés par une lettre (mois considéré) et un chiffre correspondent aux tempêtes de surô̂t.

La fonction F2 s'apparente davantage à la dynamique morphologique du bas de plage. Souvent les deux fonctions se comportent inversement : lors des événements de tempête $\mathrm{J} 1$ et F3 à F6, le profil s'érode et F1 croît tandis que F2 décroît symbolisant la génération d'une barre sableuse en bas de plage comme l'indique le schéma D (figure 9). Les événements J1 et F3 se cumulent à des marées de vives eaux portant l'excursion tidale jusqu'au pied du domaine supralittoral. En outre, l'événement M1 contribue à la fois aux décroissances de F1 et F2 lesquelles sont liées à l'accumulation de matériel en haut de plage certainement par transport éolien, à l'érosion de la mi-plage et la formation d'une barre en bas d'estran. Cette orientation du profil de plage consécutive à M1 est engendrée par les coefficients de marée dégressifs pendant la tempête (75 à 36). Ainsi seule la mi- plage est mise en mouvement et les transferts sédimentaires s'opèrent sur une zone intertidale plus courte. Les événements successifs de fin avril et début mai notés A3 et M1b sont matérialisés par l'ascension de F1. Ce sont les derniers coups de vent de l'hiver 02/03 avant l'établissement du profil saisonnier estival.

Ainsi la plage de Porsmilin affiche deux modes morphologiques à l'échelle saisonnière : en hiver, un profil aplani ; en été, une berme très développée. La dynamique du bas d'estran vient se superposer, avec une barre se formant suite aux événements tempétueux, indifféremment selon les coefficients de marée.

\subsection{Corsen}

L'ACP menée sur les profils de Corsen1 met en évidence deux nouvelles composantes G1 et G2 comptant respectivement pour 50 et $37 \%$ de la variabilité des données. G1 croît et décroît au rythme de l'engraissement et du démaigrissement du profil (fig.10B), G2 s'attache plus précisément à qualifier la dynamique du bas de plage. 

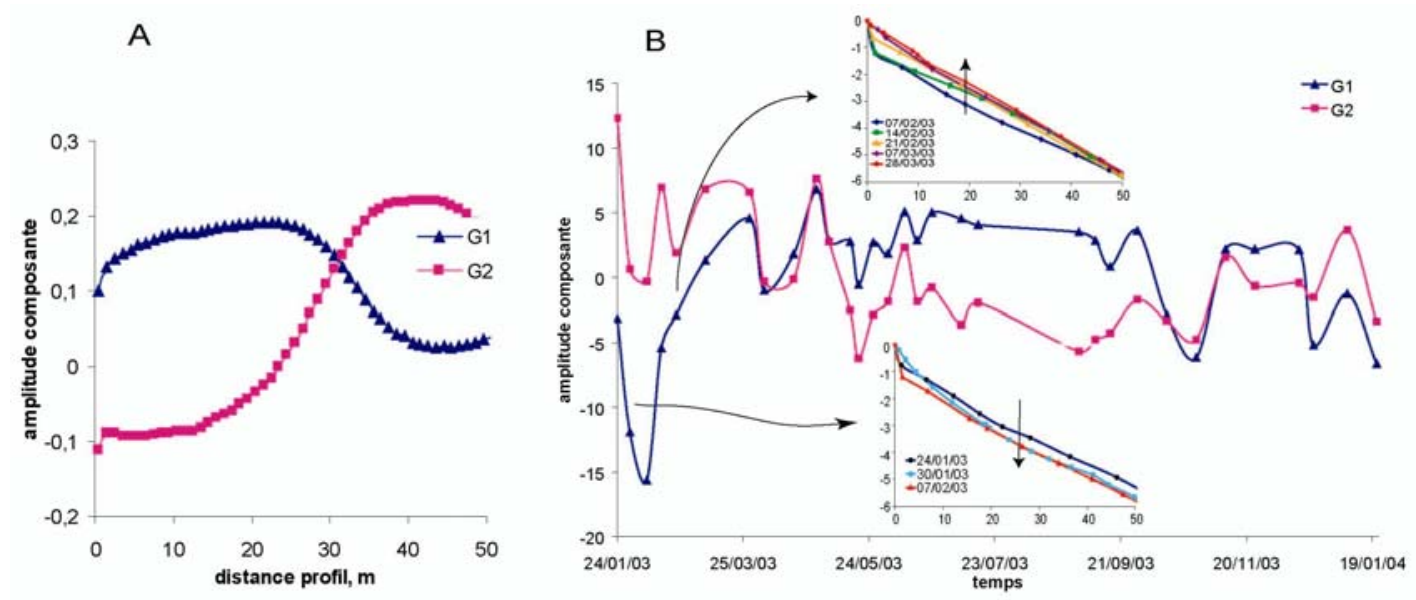

Figure 10 (A) Composantes $G 1$ et $G 2$ dans leur dimension spatiale le long du profil 1

(B) dans leur dimension temporelle.

Les variations de la composante G1 sont complétées par les profils de janvier à mars 2003.

\section{Conclusion}

L'analyse en composantes principales est un outil adapté à la compréhension de la morphodynamique des plages de Porsmilin et Corsen. Néanmoins les limitations relevées par la méconnaissance du domaine subtidal doivent être comblées. Les levés bathymétriques réalisés sur la plage de Porsmilin depuis septembre 2003 doivent aller dans le sens d'une meilleure appréhension de la morphodynamique de ces sites à une échelle spatiale satisfaisante.

\section{Références}

1.Winant, C.D., Inman, D.L. and Nordstrom, C.E. (1975) Description of seasonal beach changes using empirical eigenfunctions. Journal of Geophysical Research, 80 (15), 19791986.

2.Aubrey, D.G. (1979) Seasonal patterns of onshore/offshore sediment movement. Journal of Geophysical Research, 84 (C10), 6347-6354.

3.Larson, M., and Kraus, N.C. (1994) Temporal and spatial scales of beach profile change, Duck, North Carolina. Marine Geology, 117, 75-94.

4.Masselink, G., and Short, A.D. (1993) The effect of tide range on beach morphodynamics and morphology: a conceptual beach model. Journal of Coastal Research, 9(3), 785-800.

5.Wright, L.D., and Short, A.D. (1984) Morphodynamic variability of surf zones and beaches: a synthesis. Marine Geology, 56, 93-118.

6.Dean, R.G. (1973) Heuristic models of sand transport in the surf zone. Conference on Engineering dynamics in the coastal zone, p. 208-214.

7.Sunamura, T. (1988) Beach morphologies and their change. In K. Horikawa, Ed. Nearshore dynamics and coastal processes, p. 135-161. University of Tokyo press.

8.Guza, R.T., and Inman, D.L. (1975) Edge waves and beach cusps. Journal of Geophysical Research, 80, 2997-3012.

9.Battjes, J.A. (1974) Surf similarity. In American Society of Civil Engineers, Ed. 14th International Conference of Coastal Engineering, p. 466-480.

10.Gracia, V., Jimenez, J.A. and Sanchez-Arcilla, A. (1995) Nearshore profiles along the Ebro delta coast. Implications for coastal processes. In E. Ozhan, Ed. MEDCOAST' 95, p. 1131-1143, Tarragona, Spain. 